\title{
Elephants can always remember: Exact long-range memory effects in a non-Markovian random walk
}

\author{
Gunter M. Schütz* \\ Institut für Festkörperforschung, Forschungszentrum Jülich, D-52425 Jülich, Germany \\ Steffen Trimper \\ Fachbereich Physik, Martin-Luther-Universität, D-06099 Halle, Germany
}

(Received 24 June 2004; published 13 October 2004)

\begin{abstract}
We consider a discrete-time random walk where the random increment at time step $t$ depends on the full history of the process. We calculate exactly the mean and variance of the position and discuss its dependence on the initial condition and on the memory parameter $p$. At a critical value $p_{c}^{(1)}=1 / 2$ where memory effects vanish there is a transition from a weakly localized regime [where the walker (elephant) returns to its starting point] to an escape regime. Inside the escape regime there is a second critical value where the random walk becomes superdiffusive. The probability distribution is shown to be governed by a non-Markovian FokkerPlanck equation with hopping rates that depend both on time and on the starting position of the walk. On large scales the memory organizes itself into an effective harmonic oscillator potential for the random walker with a time-dependent spring constant $k=(2 p-1) / t$. The solution of this problem is a Gaussian distribution with time-dependent mean and variance which both depend on the initiation of the process.
\end{abstract}

DOI: 10.1103/PhysRevE.70.045101

Memory effects in non-Markovian stochastic processes are often incorporated heuristically on a coarse-grained scale into time evolution equations for physical observables, recently discussed in [1-3]. A fundamental concept of a nonMarkovian process is the continuous-time random walk introduced some years ago [4]. This theory has numerous important applications, studied for instance in [5] or recently in [6]. A broad variety of examples in biology is analyzed in [7]. Alternatively, one may derive a formally exact evolution equation for an observable by a projection mechanism [8,9] and then apply some approximation scheme for the solution of the equation. Techniques of this type are used in the context of diffusive dynamics where memory effects may lead to anomalous diffusion or even localization. In recent studies this has been demonstrated within a one-loop renormalization group approach [10], by other analytical studies [11] and confirmed by numerical methods [12]. A more fundamental approach to anomalous diffusion based on a nonequilibrium statistical description is already discussed in [13]. While very successful both phenomenologically and in predicting interesting new memory effects [14], two intrinsic shortcomings of these traditional approaches deserve attention: There is no quantitative control over the error induced by approximations, and the microscopic origin of the memory term is frequently obscured. In particular, there is usually no simple transition from an explicit and physically motivated nonMarkovian noise term to an associated non-Markovian evolution equation for the probability density which is often employed in the framework described above [15]. It is the aim of this paper to skirt these problems in the investigation of memory effects in diffusive motion by first defining a simple "microscopic" non-Markovian stochastic dynamics

\footnotetext{
*Electronic address: g.schuetz@fz-juelich.de

†Electronic address: trimper@physik.uni-halle.de
}

PACS number(s): 05.70.Ln, 05.40.-a, 87.23.Kg, 02.30.Ks

for the random walk on lattice scale, then passing to an evolution equation for the probability distribution (in formal analogy to passing to a Fokker-Planck equation (FPE) in the case of Markov processes) and finally coarse graining by taking the limit of large space and time scales. As the result we get a FPE with a time-dependent drift term. Recently stochastic processes leading to FPE with time-dependent coefficients, are discussed by several authors [18-20]. In view of those more heuristic approaches our model yields a more microscopic foundation for a special realization of a FPE with a time-dependent term.

Specifically we are interested in conditions under which an unbounded memory can induce qualitative changes in the distribution of the position as compared to the Markovian case with Gaussian distribution on large space and time scales. It is well known from the self-avoiding walk (which is a rare example for a random walk with unbounded memory where detailed exact results are known $[16,17]$ ) that the memory of the previously visited sites changes the scaling behavior of the distribution and leads to a superdiffusive mean square displacement. Here we investigate how a very different unbounded memory affects the random walk statistics and induces a transition to superdiffusive behavior. For definiteness and simplicity of notation we mainly consider a one-dimensional random walk $X_{t} \in \mathbb{Z}$ on the infinite lattice. The random walk starts at some specific point $X_{0}$ at time $t_{0}$ $=0$ and has a complete memory of its whole history. In allusion to the traditional saying that elephants can always remember, we shall refer to the random walker as elephant. In each discrete time step the elephant moves one step to the right or left, respectively (simple random walk), so the stochastic evolution equation is given by

$$
X_{t+1}=X_{t}+\sigma_{t+1},
$$

where $\sigma_{t+1}= \pm 1$ is a random variable. The memory consists of the set of random variables $\sigma_{t^{\prime}}$ at previous time steps which the elephant remembers as follows: 
(D1) At time $t+1$ a number $t^{\prime}$ from the set $\{1,2, \ldots, t\}$ is chosen randomly with uniform probability $1 / t$.

(D2) $\sigma_{t+1}$ is determined stochastically by the rule

$$
\sigma_{t+1}=\sigma_{t^{\prime}} \quad \text { with probability } p \text { and }=-\sigma_{t^{\prime}} \quad \text { with } 1-p \text {. }
$$

At the first time step $t=1$ the process is initiated as follows:

(D3) The elephant starting at $X_{0}$ moves to the right with probability $q$ and to the left with probability $1-q$, i.e.,

$$
\sigma_{1}=1 \text { with probabilty } q \text { and }=-1 \text { with } 1-q \text {. }
$$

It is obvious from the definition that

$$
X_{t}=X_{0}+\sum_{t^{\prime}=1}^{t} \sigma_{t^{\prime}}
$$

The question is to which extent the memory of the history influences the distribution of the particle position. For $p<1 / 2$ the elephant behaves metaphorically speaking like a dedicated (but not very stringent) reformer: At each step he is preferentially doing the opposite of what he (randomly) remembers to have been decided in the past. For $p>1 / 2$ the elephant is a more traditional type; he preferentially sticks to his former decision. Notice that three special cases of our model are trivial: (i) In the borderline case $p=1 / 2$ the choice of $\sigma_{t+1}$ is \pm 1 with equal probability, no matter what the history was. Hence one has the standard Markovian random walk, which converges to Brownian motion on large scales. In this case the initial parameter $q$ plays only a marginal role with no macroscopic significance. (ii) In the limiting case $p=1$ the dynamics become essentially deterministic. Given the first decision (which is random), the elephant moves with probability 1 (ballistically) always one step in the same direction as in the first move. Hence the first step is macroscopically relevant. (iii) For $q=1 / 2$ the mean position of the elephant is zero for all $p$. Nevertheless the distribution of $X_{t}$ depends nontrivially on $p$.

To study the mean position $\left\langle X_{t}\right\rangle$ we first note that given the previous history $\left\{\sigma_{1}, \ldots, \sigma_{t}\right\}$ one has the conditional probability that the increment $\sigma_{t+1}$ takes the value $\sigma= \pm 1$

$P\left[\sigma_{t+1}=\sigma \mid \sigma_{1}, \ldots, \sigma_{t}\right]=\frac{1}{2 t} \sum_{k=1}^{t}\left[1+(2 p-1) \sigma_{k} \sigma\right]$ for $t \geqslant 1$.

This follows from the definitions (D1), (D2) of the process. For $t=0$ we get in accordance with rule (D3)

$$
P\left[\sigma_{1}=\sigma\right]=\frac{1}{2}[1+(2 q-1) \sigma]
$$

Thus for $t \geqslant 1$ the conditional mean increment is given by

$$
\begin{aligned}
\left\langle\sigma_{t+1} \mid \sigma_{1} \ldots \sigma_{t}\right\rangle & =\sum_{\sigma= \pm 1} \sigma P\left[\sigma_{t+1}=\sigma \mid \sigma_{1}, \ldots, \sigma_{t}\right] \\
& =\frac{2 p-1}{t}\left(X_{t}-X_{0}\right) .
\end{aligned}
$$

These relations form the basis of the subsequent analysis of the process. Below we shall frequently use the shifted parameters

$$
\alpha=2 p-1, \quad \beta=2 q-1,
$$

which are in the range $[-1,1]$. Negative $\alpha$ corresponds to the "reformer," positive $\alpha$ parametrizes the "traditionalist"elephant. The effectively memoryless Markovian case is $\alpha=0$. From (7) the conventional mean value (obtained by summing over all previous realizations of the process) is given by

$$
\left\langle\sigma_{t+1}\right\rangle=\frac{\alpha}{t}\left(\left\langle X_{t}\right\rangle-X_{0}\right)
$$

and gives rise to the recursion for the mean displacement $\left\langle x_{t}\right\rangle=\left\langle X_{t}\right\rangle-X_{0}$

$$
\left\langle x_{t+1}\right\rangle=\left(1+\frac{\alpha}{t}\right)\left\langle x_{t}\right\rangle \text { for } t \geqslant 1 .
$$

For the first time step one has $\left\langle x_{1}\right\rangle=\left\langle\sigma_{1}\right\rangle=2 q-1=\beta$. The solution of (10) is obtained by iteration

$$
\left\langle x_{t}\right\rangle=\left\langle\sigma_{1}\right\rangle \frac{\Gamma(t+\alpha)}{\Gamma(\alpha+1) \Gamma(t)} \sim \frac{\beta}{\Gamma(\alpha+1)} t^{\alpha} \quad \text { for } \quad t \gg 1
$$

For $\alpha<0$ (reformer) the mean displacement vanishes for large $t$ algebraically, the elephant stays on average essentially where it started. For $\alpha>0$ (traditionalist) the mean displacement increases indefinitely, albeit with decreasing velocity. The direction of the escape from the starting position is determined by the first (random) decision. If the first move is positive, the average direction of motion is to the right. Otherwise the elephant moves on average to the left. At the transition point $\alpha_{c}^{(1)}=0$ the mean displacement is independent of time, as is known for the usual Markovian random walk. Recursion relations for higher-order moments also follow straightforwardly from (5). They obey recursions of the form

$$
M_{t+1}=f_{t}+g_{t} M_{t} \text { for } t \geqslant 1,
$$

where $M_{t}$ is some moment and $f_{t}, g_{t}$ are known functions, related to lower moments. The general solution of (12) is given by

$$
M_{t}=M_{1} \prod_{k=1}^{t-1} g_{k}+\sum_{n=1}^{t-1}\left[f_{n} \prod_{k=n+1}^{t-1} g_{k}\right]
$$

which is easily verified. In particular for the second moment of the displacement one finds the recursion

$$
\left\langle x_{t+1}^{2}\right\rangle=1+\left(1+\frac{2 \alpha}{t}\right)\left\langle x_{t}^{2}\right\rangle
$$

Using (13) it is solved by 


$$
\left\langle x_{t}^{2}\right\rangle=\frac{t}{2 \alpha-1}\left[\frac{\Gamma(t+2 \alpha)}{\Gamma(t+1) \Gamma(2 \alpha)}-1\right] .
$$

We first notice that the mean square displacement does not depend on the initial decision parametrized by $q$ since $\left\langle x_{1}^{2}\right\rangle$ $=1$ for any $q$. Asymptotically one has

$$
\begin{gathered}
\left\langle x_{t}^{2}\right\rangle=\frac{t}{3-4 p}, \quad p<3 / 4 ; \quad\left\langle x_{t}^{2}\right\rangle=t \ln t, \quad p=3 / 4 ; \\
\left\langle x_{t}^{2}\right\rangle=\frac{t^{4 p-2}}{(4 p-3) \Gamma(4 p-2)}, \quad p>3 / 4 .
\end{gathered}
$$

Before discussing this result we remind the reader that the displacement $x_{t}=X_{t}-X_{0}$ refers to the displacement from the initial position, not to the displacement of the actual position from its mean. Remarkably there is no qualitative change at $\alpha_{c}^{(1)}=0$ where the transition to the escape regime occurs, yet there are two distinct regimes inside the escape regime.

(1) For $\alpha<1 / 2$ (corresponding to $p<3 / 4$ ) the mean square displacement increases asymptotically linearly in time. Hence the localized regime $\alpha<\alpha_{c}^{(1)}=0$ corresponds to a weak localization in the sense that the initial mean displacement vanishes for large $t$, but the variance increases diffusively with a diffusion coefficient $D=1 /(6-8 p)$. In the range $0 \leqslant \alpha<1 / 2$ (corresponding to $1 / 2 \leqslant p<3 / 4$ ) the mean displacement diverges (escape regime), but with an exponent $\alpha<1 / 2$. Therefore, the mean square displacement is still larger than the square of the mean and the variance $\left\langle x_{t}^{2}\right\rangle-\left\langle x_{t}\right\rangle^{2}$ remains diffusive.

(2) For $\alpha>1 / 2$ (corresponding to $p>3 / 4$ ) the mean square displacement increases stronger than linearly $\sim t^{4 p-2}$ and is of the same order as the square of the mean, but with a different prefactor. Hence the variance becomes superdiffusive with an effective diffusion coefficient depending both on time and on $q$.

(3) At the critical value $\alpha_{c}^{(2)}=1 / 2$ (corresponding to $p$ $=3 / 4$ ) the right-hand side of (16) reduces to $\sum_{n=1}^{t} t / n$ $\sim t \ln t$. The elephant is marginally superdiffusive.

The results of the previous section are sufficient for the characterization of the large scale walk properties of the elephant only if the increments $\sigma_{n}$ are independent random variables, i.e., for $\alpha=0$. In this case the central limit theorem guarantees convergence of the distribution of $X_{t}$ to a Gaussian. In order to obtain information about the distribution for $\alpha \neq 0$ we consider the complex-valued characteristic function $Q_{t}(k)=\left\langle e^{i k x_{t}}\right\rangle$. Using (5) it obeys the equation

$$
\left\langle Q_{t+1}(k)\right\rangle=\cos k Q_{t}(k)+\frac{\alpha}{t} \sin k \frac{d}{d k} Q_{t}(k) .
$$

The Fourier transform $P_{t}(x)$ is the probability that the displacement at time $t$ takes the value $x$. This is equal to the conditional probability $P\left(Y, t \mid X_{0}, 0\right)$ that the position $X$ of the elephant at time $t$ equals $Y=X_{0}+x$, given that it started at $X_{0}$ at $t=0$. From (17) we find a discrete evolution equation formally analogous to the FPE for usual random walks,

$$
\begin{aligned}
P\left(Y, t+1 \mid X_{0}, 0\right)= & \frac{1}{2}\left[1-\frac{\alpha}{t}\left(Y-X_{0}+1\right)\right] P\left(Y+1, t \mid X_{0}, 0\right) \\
& +\frac{1}{2}\left[1+\frac{\alpha}{t}\left(Y-X_{0}-1\right)\right] P\left(Y-1, t \mid X_{0}, 0\right) .
\end{aligned}
$$

This equation (valid for $t \geqslant 1$ ) may be interpreted in terms of a time-inhomogeneous random walk which does not memorize its full history, but only its initial position at time $t=0$. It describes a hopping process where in each step the walker at position $Y$ hops to the right with probability $p_{r}=[1+\alpha(Y$ $\left.\left.-X_{0}\right) / t\right] / 2$ and to the left with probability $p_{l}=[1-\alpha(Y$ $\left.\left.-X_{0}\right) / t\right] / 2$, respectively. At first sight these stochastic dynamics look like a time-inhomogeneous Markov chain where $X_{0}$ is some parameter. However, we stress that in (18) the quantity $X_{0}$ is not a parameter, but the initial position of the elephant. The hopping probabilities implicit in (18) are not valid for an elephant starting at a position different from $X_{0}$ or which starts at $X_{0}$ at a later time $t>0$. The non-Markovian character of the dynamics is expressed in the fact that the evolution equation (18) is different for each initial position; see [15] for a general discussion of similar non-Markovian evolution equations. The qualitative features of the elephant which became apparent through the study of its mean position are expressed in the hopping probabilities $p_{r, l}$. For positive $\alpha$ the local bias

$$
b(x, t)=p_{r}-p_{l}=\frac{\alpha x}{t}
$$

is positive for positive displacement, hence the particle on average escapes. On the other hand, for negative $\alpha$, the bias is opposite to the actual displacement, reminiscent of some effective restoring force. This becomes very transparent in the continuum limit (large displacement $x$ and time $t$ ). In terms of $x, t$ (18) takes the form

$$
\frac{\partial P(x, t)}{\partial t}=\frac{1}{2} \frac{\partial^{2}}{\partial x^{2}} P(x, t)-\frac{\alpha}{t} \frac{\partial}{\partial x}[x P(x, t)], \quad t>0
$$

of a FPE for a Brownian particle in a harmonic oscillator potential with spring constant $k=\alpha / t$. The last relation is a special case of more general FPE with time-dependent coefficients that has been investigated in several papers [18-20]. Whereas the approach in those papers is phenomenologically, we demonstrate in the frame of a microscopic model the origin of such FPE. From Eq. (20) one obtains recursion relations for the moments of the distribution. Let us denote the even and the odd moments by

$$
a_{n}(t)=\left\langle x^{2 n}\right\rangle \quad b_{n}(t)=\left\langle x^{2 n+1}\right\rangle \quad \text { with } n=0,1,2, \ldots .
$$

Using Eq. (20) we obtain for the even moments

$$
\frac{d}{d t} a_{n}(t)-\frac{2 n \alpha}{t} a_{n}(t)=n(2 n-1) a_{n-1}(t),
$$

and a similar equation for the odd moments. In particular, we have 


$$
\frac{d}{d t}\langle x\rangle=\frac{\alpha}{t}\langle x\rangle
$$

with the solution

$$
\langle x(t)\rangle=\left\langle x\left(t_{0}\right)\right\rangle\left(\frac{t}{t_{0}}\right)^{\alpha} \equiv \bar{x}(t) \quad \text { with } \quad t \geqslant t_{0}>0,
$$

in agreement with (11). Here $t_{0}$ is the temporal cutoff scale, reflecting the breakdown of the continuum approximation for $t \rightarrow 0$. For second moment we find

$$
\left\langle x^{2}\right\rangle=\Delta(t)+\bar{x}^{2}(t)
$$

with $\bar{x}(t)$ given by (24) and

$$
\Delta(t)=\left(\frac{t}{t_{0}}\right)^{2 \alpha}\left[\left\langle x^{2}\left(t_{0}\right)\right\rangle-\bar{x}^{2}\left(t_{0}\right)\right]+\frac{t}{2 \alpha-1}\left[\left(\frac{t}{t_{0}}\right)^{2 \alpha-1}-1\right] .
$$

Since the initial distribution is assumed to be concentrated at $x_{0}$ the initial variance and so the first term in (26) vanishes. Thus we can read off the effective diffusion coefficient

$$
D(t)=\frac{1}{4 \alpha-2}\left[\left(\frac{t}{t_{0}}\right)^{2 \alpha-1}-1\right]
$$

of the elephant. In (22) one recognizes the recursion relations for the moments of a Gaussian distribution. Indeed, one can straightforwardly verify that

$$
P(x, t)=\frac{1}{\sqrt{4 \pi t D(t)}} \exp \left\{-\frac{[x-\bar{x}(t)]^{2}}{4 t D(t)}\right\}
$$

solves the evolution equation (17) for the initial condition $\delta\left(Y-X_{0}\right)=\delta\left[x-\bar{x}\left(t_{0}\right)\right]$. The centered even moments

$$
M_{2 n}=\left\langle[x-\bar{x}(t)]^{2 n}\right\rangle=(2 n-1) ! ![2 t D(t)]^{n},
$$

which satisfy the recursion relation (22) are given by the usual expression for a Gaussian distribution.

The result can be generalized to the $d$-dimensional case with a separate memory for each space direction. To this aim the rules (D1)-(D3) are generalized accordingly. Equation (4) is changed to an equation for a $d$-dimensional vector and correspondingly the conditional probability (18) depends also on the $d$-dimensional position vector. In the continuous limit the evolution equation reads

$$
\frac{\partial P(\vec{x}, t)}{\partial t}=\frac{1}{2} \nabla^{2} P(\vec{x}, t)-\frac{\alpha}{t} \nabla[\vec{x} P(\vec{x}, t)] .
$$

Based on that evolution equation we find also the equations for the even and odd moments. In particular, the even moment $a_{n}(t ; d)=\left\langle\left(\vec{x}^{2}\right)^{n}\right\rangle$ satisfies instead of Eq. (22) the equation

$$
\frac{d}{d t} a_{n}(t ; d)-\frac{2 n \alpha}{t} a_{n}(t ; d)=n[d+2(n-1)] a_{n-1}(t ; d) .
$$

A solution of this equation that yields the even centered moments is

$$
a_{n}(t ; d)=\frac{\Gamma\left(\frac{d}{2}+n\right)}{\Gamma\left(\frac{d}{2}\right)}[2 t D(t)]^{n} .
$$

The odd moments $b_{n}^{\beta}(t ; d)=\left\langle\left(\vec{x}^{2}\right)^{n} x_{\beta}\right\rangle$ obey the equation

$$
\frac{d}{d t} b_{n}^{\beta}(t ; d)-\frac{(2 n+1) \alpha}{t} b_{n}^{\beta}(t ; d)=n(2 n+d) b_{n-1}^{\beta}(t ; d)
$$

for the odd moments of a $d$-dimensional Gaussian distribution.

Starting with a microscopic model of a random walk with unbounded long-time memory (the "elephant"), we have calculated the exact mean and variance, respectively, as well as the single-time probability distribution for the position of the elephant on large scales. Surprisingly the memory effects incorporated in the probability distribution at time $t$ amount to a time-inhomogeneous random walk where only the initial position and starting time play a role.

This work was supported by the DFG (SFB 418).
[1] L. S. Tsimring and A. Pikovsky, Phys. Rev. Lett. 87, 250602 (2001).

[2] C. Masoller, Phys. Rev. Lett. 88, 034102 (2002); 90, 020601 (2003).

[3] M. Freeman, Nature (London) 408, 313 (2000).

[4] E. W. Montroll and G. H. Weiss, J. Math. Phys. 6, 167 (1965).

[5] D. ben-Avraham and S. Havlin, Diffusion and Reactions in Fractals and Disordered Systems (Cambridge University Press, Cambridge, 2000).

[6] K. Ito and S. Miyazaki, Prog. Theor. Phys. 110, 875 (2003).

[7] J. D. Murray, Mathematical Biology, Part I (Springer, Berlin, 2000).

[8] R. Zwanzig, Phys. Rev. 124, 983 (1961).

[9] H. Mori and H. Fujisaka, Prog. Theor. Phys. 49, 764 (1973).

[10] M. Schulz and S. Stepanow, Phys. Rev. B 59, 13528 (1999).

[11] M. Schulz and S. Trimper, Phys. Rev. B 64, 233101 (2001).
[12] B. M. Schulz, M. Schulz, and S. Trimper, Phys. Rev. E 66, 031106 (2002).

[13] K. G. Wang and M. Tokuyama, Physica A 265, 341 (1999).

[14] S. Trimper, K. Zabrocki, and M. Schulz, Phys. Rev. E 66, 026114 (2002).

[15] P. Hänggi and H. Thomas, Z. Phys. B 26, 85 (1977).

[16] N. Madras and G. Slade, The Self-Avoiding Walk (Birkhäuser, Boston, 1993).

[17] G. F. Lawler, O. Schramm, W. Werner, e-print math.PR/ 0204277.

[18] F. Lillo and R. N. Mantegna, Phys. Rev. E 61, R4675 (2000).

[19] S. I. Denisov and W. Horsthemke, Phys. Rev. E 62, 7729 (2000).

[20] L. C. Malacarne, R. S. Mendes, I. T. Pedron, and E. K. Lenzi, Phys. Rev. E 65, 052101 (2002). 